\title{
Aerodynamic sound generation by global modes in hot jets
}

\author{
LUTZ LESSHAFFT ${ }^{1} \uparrow$, PATRICK HUERRE ${ }^{1}$ \\ AND PIERRE SAGAUT ${ }^{2}$ \\ ${ }^{1}$ Laboratoire d'Hydrodynamique, CNRS - École Polytechnique, 91128 Palaiseau, France \\ ${ }^{2}$ D'Alembert Institute, Université Pierre et Marie Curie (Paris 6), Boite 162, 4 place Jussieu, \\ 75252 Paris Cedex 05, France
}

(Received 29 July 2009; revised 17 November 2009; accepted 20 November 2009)

The acoustic field generated by the synchronized vortex street in self-excited hot subsonic jets is investigated via direct numerical simulation of the compressible equations of motion in an axisymmetric geometry. The simulation simultaneously resolves both the aerodynamic near field and the acoustic far field. Self-sustained near-field oscillations in the present flow configurations have been described as nonlinear global modes in an earlier study. The associated acoustic far field is found to be that of a compact dipole, emanating from the location of vortex roll-up. A far-field solution of the axisymmetric Lighthill equation is derived, on the basis of the source term formulation of Lilley (AGARD-CP, vol. 131, 1974, pp. 13.1-13.12). With the near-field source distributions obtained from the direct numerical simulations, the Lighthill solution is in good agreement with the far-field simulation results. Fluctuations of the enthalpy flux within the jet are identified as the dominant aeroacoustic source. Superdirective effects are found to be negligible.

\section{Introduction}

Subsonic jets, if they are sufficiently hot compared with the ambient air, may bifurcate to a regime of intrinsic self-sustained oscillations that give rise to a street of highly regular ring vortices. This oscillator-type behaviour in hot jets has first been observed experimentally by Monkewitz et al. (1990). Recent numerical studies (Lesshafft et al. 2006; Lesshafft, Huerre \& Sagaut 2007) have demonstrated that these oscillations are due to an absolute instability of the jet profile near the nozzle and that they may be described theoretically as a nonlinear global mode.

The present study examines the acoustic far field that is radiated from the self-sustained vortex street in globally unstable hot jets. The thick-shear-layer configurations treated in Lesshafft et al. (2006), with the ambient-to-jet temperature ratios $S=0.1,0.2$ and 0.3 , are chosen for this investigation. Although only the aerodynamic near-field dynamics have been addressed in our previous publications, the computational domain of the direct numerical simulation (DNS) already encompassed a large portion of the acoustic far field. These far-field results are now used to analyse the directivity pattern and the physical sound generation mechanisms that dominate the acoustic radiation from the global mode in a hot jet. 
Numerical simulations in which the acoustic field is computed from first principles have become known as direct noise calculations (DNC). Mitchell, Lele \& Moin (1999) were the first to apply this approach to the jet noise problem. Their study focused on the sound generated by the pairing of large-scale vortices in forced isothermal jets. As in the present paper, the simulations of Mitchell et al. (1999) were carried out in an axisymmetric setting, which hindered the onset of turbulence and thus allowed a study of the isolated aeroacoustic behaviour of large-scale near-field dynamics. Freund (2001) performed direct numerical simulations resolving the acoustic field of a three-dimensional turbulent isothermal jet at low Reynolds number, which gave results in good agreement with experimental measurements. The low-Reynoldsnumber restriction was relaxed by Bogey, Bailly \& Juvé (2003) through the use of large-eddy simulation (LES) subgrid modelling. In subsequent papers (Bogey \& Bailly 2004, 2005), these authors investigated the influence of numerical boundary conditions and various subgrid models, as well as Mach and Reynolds number effects. A list of further LES studies that have directly resolved the acoustic field of turbulent jets is given in Bodony \& Lele (2006). Only few among these include the effect of jet heating: the simulations of Andersson, Eriksson \& Davidson (2005) successfully reproduced experimental acoustic measurements of Jordan et al. (2002) in a subsonic hot jet when the nozzle geometry was included. Shur, Spalart \& Strelets (2005) achieved good agreement with the reference experiments of Tanna (1977) and Viswanathan (2004) in hot-jet simulations in both subsonic and supersonic settings.

The large majority of numerical jet noise studies, as reviewed by Wang, Freund \& Lele (2006), relies on hybrid methods. Based on jet near-field data obtained from Reynolds-averaged Navier-Stokes or LES calculations, the acoustic far field is calculated according to an acoustic analogy. The common objective of these investigations is to validate the predictive capabilities of acoustic analogies or boundary integral methods, by comparison with available acoustic data from experiments or direct calculations. In some instances, computed near-field data have also been used to investigate the acoustic source mechanisms underlying the far-field spectrum and directivity pattern: Mitchell et al. (1999) compared the relative importance of individual source terms, in the sense of Lighthill's equation, in forced laminar jets. Their results demonstrated that streamwise variations of the source strength, even in regions of very low amplitude, greatly influence the far-field sound directivity. The analysis of Freund (2001) of DNS data for a turbulent unheated jet identified large-scale structures as the dominant noise sources. The spatial distribution of the structures takes the form of a wave packet, similar to what the instability theory would predict for a laminar setting.

The numerical analysis of acoustic source mechanisms in hot jets, despite their practical importance, has received little attention in the recent literature. Fortune \& Gervais (1999) proposed a prediction scheme for temperature-related turbulence noise, on the basis of the $k-\epsilon$ model. In LES studies by Bodony \& Lele (2005) of hot turbulent jets in the high subsonic and supersonic regime, the sound field was computed directly. In the framework of Lighthill's acoustic analogy, cancellation effects between Reynolds stress and so-called entropy contributions were documented. Similar cancellation effects at a high subsonic Mach number were reported by Lew, Blaisdell \& Lyrintzis (2007), by means of a hybrid numerical approach. Lew et al. (2007) concluded from their results that the sound field of a hot jet at low Mach number is strongly dominated by radiation from entropy-related sources. 
The present study investigates sound generation mechanisms due to large-scale instability structures (global modes) in self-excited hot jets at low Mach number. The numerical approach is similar to that of Mitchell et al. (1999): the axisymmetric equations of motion are resolved directly in the near and far fields. This axisymmetric restriction prevents the development of small-scale turbulence; it is justified by the experimental observations of Monkewitz et al. (1990). Lighthill's equation is used not to predict the far-field sound, but as an analytical tool, in order to identify dominant source mechanisms. To this end, the aeroacoustic source terms of the Lighthill equation are recast in the formulation proposed by Lilley $(1974,1996)$. Lighthill's original formulation includes one monopole source term that is commonly linked to entropy fluctuations in the near field, although it is known to contain both isentropic and non-isentropic components (see for instance A. Michalke's note in Lilley 1974). This source term will be referred to as the 'excess density' (Dowling 1992) in the following. Lilley's formulation decomposes the excess-density term into explicit monopole and dipole sources, and it allows for a less ambiguous interpretation of the sound-producing physical mechanisms, as will be demonstrated in the current paper. Freund (2003) achieved an improved interpretation of his 2001 simulation results based on Lilley's source decomposition. Similarly, Bodony \& Lele (2008) used Lilley's formalism for a refined analysis of some of their earlier results (Bodony \& Lele 2005), including one case of a transonic hot jet.

Laufer \& Yen (1983) measured the acoustic radiation due to regular vortex pairing events in forced isothermal jets at low Mach number. The acoustic field was found to exhibit a 'superdirective' beaming pattern, with maximum intensity $I$ at the radiation angle $\vartheta=0$ in the downstream direction of the jet:

$$
I(\vartheta) \propto \exp \left[-A\left(1-M a_{c v} \cos \vartheta\right)^{2}\right], \quad A=45,
$$

where the Mach number $M a_{c v}$ is formed with the vortex convection velocity. This result has been confirmed in only one experimental configuration by Fleury, Bailly \& Juvé (2005). According to the discussion of Laufer \& Yen (1983), such strong beaming behaviour seemed unlikely to arise from an acoustically compact source region: the near-field fluctuation amplitudes associated with vortex pairing were measured to vary as a Gauß function in the streamwise direction, with a half-width an order of magnitude smaller than the acoustic wavelength. However, the theoretical analyses of Huerre \& Crighton (1983) and Crighton \& Huerre (1990) have demonstrated that a perfectly Gaussian shape of the near-field wave packet indeed results in an antenna factor of the form (1.1). More generally, these authors surmised that any extended wave packet, depending on its precise envelope shape, may emit a superdirective sound field. The nonlinear global modes in hot jets described in Lesshafft et al. (2006, 2007) may be represented as such extended wave packets. As their spatial amplitude and phase modulations are precisely known from the numerical simulations, the approach of Huerre \& Crighton (1983) will be applied in the present study to the case of a globally unstable hot jet. It is hoped that this analysis will further elucidate the conditions for superdirective sound radiation from low-Mach-number jets.

The paper is organized as follows. The flow parameters are defined, and the numerical methods used in the direct computations are outlined in $\S 2.1$. Simulation results in the acoustic far field are presented in $\S 2.2$. In $\S 3$, the solution procedure for the Lighthill equation is laid out. This formalism is then applied to three hot-jet configurations in $\S 4$, and the main conclusions are summarized in $\S 5$. 


\section{Direct noise computation of a hot jet}

\subsection{Flow configuration and numerical method}

The flow model and the numerical solution techniques employed in the simulation have been presented in Lesshafft et al. (2006) and are documented in full detail in Lesshafft (2006): the problem is formulated in axisymmetric coordinates $x$ and $r$; the conservative flow variables $\boldsymbol{q}=(\rho, \rho u, \rho v, \rho E)$ are decomposed into a steady baseflow component $\boldsymbol{q}_{b}$ and an unsteady perturbation component $\boldsymbol{q}^{\prime}$. The symbol $\rho$ denotes density; $u$ and $v$ are the axial and radial velocity components; $E$ is the total energy. The baseflow $\boldsymbol{q}_{b}(x, r)$ is obtained by numerical integration of the compressible boundary layer equations, starting from an analytical jet velocity profile ('profile 2' of Michalke 1984) at the upstream boundary $x=0$ of the computational domain. The temporal evolution of perturbations $\boldsymbol{q}^{\prime}(x, r, t)$ within this baseflow is then computed according to the compressible equations of continuity, momentum and energy, closed by the equation of state for a perfect gas (note that there is a typographical error in equation $2.4 b$ of Lesshafft et al. 2006, which has been corrected in Lesshafft 2006).

All quantities are made non-dimensional with respect to the jet radius $R$ and the jet centreline values of density $\rho_{c}$, velocity $U_{c}$ and temperature $T_{c}$ in the potential core. The three flow configurations investigated in this study are defined by the following parameters:

$$
\left.\begin{array}{rlrl}
R / \theta & =10, & & S=0.1,0.2,0.3, \\
R e & =1000, & & M a_{c}=0.1, \\
P r & =1, & & \gamma=1.4 .
\end{array}\right\}
$$

As defined in Lesshafft et al. (2006), Re, $M a_{c}$ and $P r$ are the Reynolds, Mach and Prandtl numbers; $\theta$ is the momentum shear layer thickness of the inlet velocity profile; $S=T_{\infty} / T_{c}$ is the ambient-to-jet temperature ratio; and $\gamma$ is the ratio of specific heats. Note that the Mach number $M a_{c}$ is defined with respect to the speed of sound on the centreline. It is easily converted to the more conventional definition $M a_{\infty}=U_{c} / c_{\infty}=S^{-1 / 2} M a_{c}$. The three temperature ratios $S=0.1,0.2$ and 0.3 then yield Mach numbers $M a_{\infty}=0.32,0.22$ and 0.18 .

Among the three jet configurations (2.1), the $S=0.3$ case will be discussed in full detail because it is trusted to be the least affected by inaccuracies due to the numerical boundary treatment. Results for the two other cases, $S=0.1$ and 0.2 , are summarized in $\S 4.2$ in order to test the validity of the main conclusions for a range of globally unstable temperature ratios and Mach numbers. In the following, through to the end of $\S 4.1$, the discussion will focus on the $S=0.3$ case.

The long-time response of a jet to an initial pulse perturbation is computed on an orthogonal grid that discretizes the physical domain $0 \leqslant r \leqslant 46$ and $0 \leqslant x \leqslant 80$ into $349 \times 801$ grid points. Outside this region, all perturbations are attenuated by artificial damping and strong grid stretching in sponge zones extending over $46<r \leqslant 200$ and $80<x \leqslant 105$. First-order characteristic boundary conditions given by Giles (1990) are applied at the upstream numerical boundary in order to minimize acoustic reflections and spurious coupling of acoustic and vortical waves in the jet shear layer. Inside the physical region of the computational domain, spatial derivatives in the governing equations are evaluated using a sixth-order explicit finite-difference scheme, and the solution is time advanced via a third-order Runge-Kutta algorithm.

\subsection{Direct numerical simulation results}

As discussed in $\S 5$ of Lesshafft et al. (2006), the near-field dynamics of jets with parameters (2.1) are characterized by self-sustained oscillations that give rise to a 
highly regular roll-up of the jet shear layer into evenly spaced ring vortices. In theoretical terms, this periodic flow state is described as a nonlinear global mode. The global frequency of vortex roll-up at $S=0.3$ has been determined to be $\omega_{g}=0.728$ in the numerical simulations ('mode 1' in Lesshafft et al. 2006). It has been discussed that the $S=0.3$ configuration represents the critical case for the marginal onset of global instability and that the asymptotic approach of the final oscillating state is extremely slow as a consequence. For the present investigation, the computations have been continued over an additional number of approximately 30 oscillation periods, and the global frequency has further converged to a value of $\omega_{g}=0.722$.

Only configurations with $R / \theta=10$ are chosen for the present investigation because the observed global mode structure is unaffected by vortex pairing; in simulations with thinner initial shear layers $R / \theta>10$ (Lesshafft et al. 2007), a secondary instability of the vortex street leads to regular 'leap-frogging' of neighbouring vortices. These events modify the spatial envelope of the fundamental global mode oscillations and at the same time radiate a subharmonic sound field. An investigation of sound generation mechanisms due to vortex pairing is not the subject of the current paper. The restriction to globally unstable configurations without vortex pairing further limits the parameter regime to low values of the Mach number and temperature ratio (see figure 10 of Lesshafft \& Huerre 2007).

In the present flow examples, the acoustic field may be regarded as monochromatic: the near field is free of random fluctuations, and as in the low-Mach-number cases of Mitchell et al. (1999), harmonic components in the far field are negligible when compared with the fundamental sound component $\left(\omega_{g}=0.722\right.$ for $\left.S=0.3\right)$. The acoustic wavelength is then $\lambda_{a}=2 \pi c_{\infty} / \omega_{g}=47.7$, which approximately corresponds to the radial extent of the physical domain in the simulation. In the following, the acoustic field is investigated in terms of the temporal Fourier coefficient of pressure fluctuations, defined as

$$
\hat{p}(\boldsymbol{x} ; \omega)=\int p^{\prime}(\boldsymbol{x}, t) \mathrm{e}^{\mathrm{i} \omega t} \mathrm{~d} t .
$$

The isocontours of $\left|\hat{p}\left(\boldsymbol{x} ; \omega_{g}\right)\right|$ are shown in figure 1 : the acoustic field is composed of two lobes, with an extinction angle at about $90^{\circ}$ from the jet axis. The apparent source location at $x=9$ corresponds to the streamwise station of vortex roll-up (compare with figure $7 a$ of Lesshafft et al. 2006). The decibel levels in figure 1 are scaled with respect to the maximum near-field pressure amplitude. Because of weak reflections from the downstream sponge region, the isocontours in the acoustic field at $x>60$ are slightly distorted and are not shown in figure 1. For a quantitative examination of the acoustic directivity pattern, values of $|\hat{p}|$ are interpolated along an arc of radius $\xi=30$ around the apparent source location. Figure 2 reveals that the directivity pattern observed in the numerical simulation closely corresponds to that of a compact dipole $\hat{p} \propto \cos \vartheta$, with the radiation angle $\vartheta$ being measured relative to the downstream jet axis (see figure 1).

The isocontour diagram in figure 1 suggests that the transition from the aerodynamic near field to the acoustic far field takes place over a length scale much shorter than the acoustic wavelength. This observation is confirmed in figure 3 , which displays the variation of $|\hat{p}|$, measured at an angle $\vartheta=60^{\circ}$, as a function of distance $\xi$ from the apparent sound source location. Outside the near field of the jet, for $\xi \gtrsim 8$, the slope of the pressure amplitude quickly adjusts to the characteristic decay rate $|\hat{p}| \propto \xi^{-1}$ of the acoustic far field. 


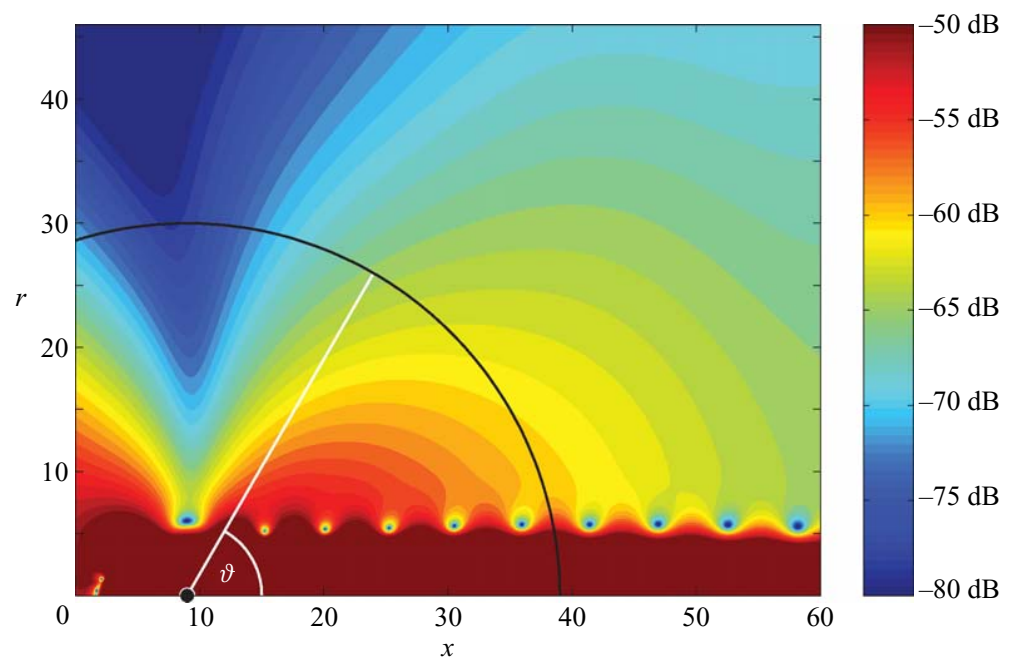

FIGURE 1. The isocontours of the pressure amplitude $\left|\hat{p}\left(r, x ; \omega_{g}\right)\right|$ in the acoustic far field of a jet with $S=0.3$. The apparent sound source on the jet axis is located at $x=9$. The directivity pattern in figures 2 and 5 is extracted along the black arc of radius 30 .

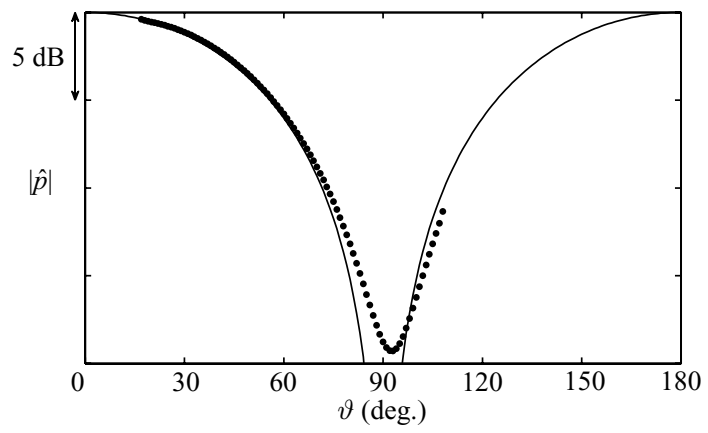

FIGURE 2. The dots denote pressure amplitude as a function of radiation angle $\vartheta$, interpolated along the arc indicated in figure 1; the line denotes directivity of a compact dipole $\hat{p} \propto \cos \vartheta$.

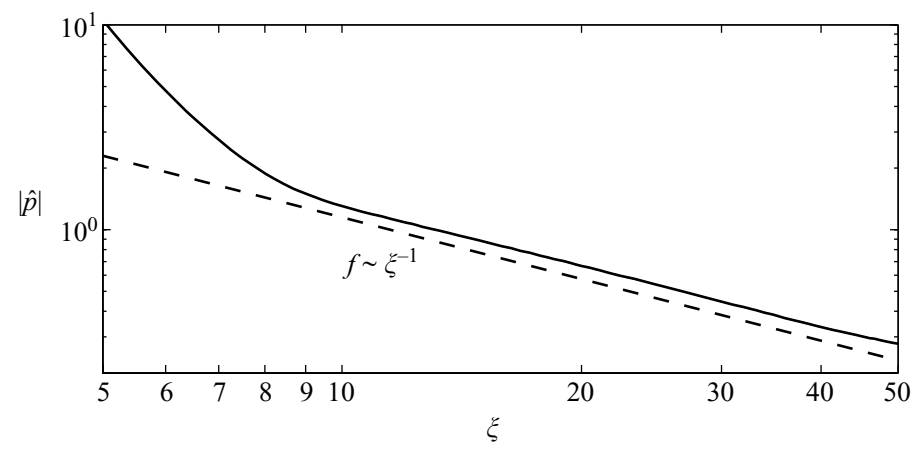

FIgURE 3. The solid line denotes pressure amplitude $|\hat{p}|$ as a function of observer distance $\xi$, measured along the radiation angle $\vartheta=60^{\circ}$. The dashed line denotes algebraic decay $\propto \xi^{-1}$ as expected in the acoustic far field. 


\section{Integration of the Lighthill equation}

\subsection{Source term decompositions of Lighthill (1952) and Lilley (1974)}

Lighthill (1952) derived an exact inhomogeneous wave equation for acoustic fluctuations by combining the continuity and momentum equations. In Cartesian coordinates $x_{i}$, the Lighthill equation for pressure fluctuations reads (see for instance Crighton 1975)

$$
\frac{1}{c_{\infty}^{2}} \frac{\partial^{2} p^{\prime}}{\partial t^{2}}-\frac{\partial^{2} p^{\prime}}{\partial x_{i}^{2}}=\frac{\partial^{2} S_{i j}}{\partial x_{i} \partial x_{j}}-\frac{\partial^{2} \rho_{e}}{\partial t^{2}},
$$

where $c_{\infty}$ denotes the speed of sound in the far field. The stress tensor

$$
S_{i j}=\rho u_{i} u_{j}-\tau_{i j}
$$

is composed of Reynolds stresses and viscous terms, while the excess density

$$
\rho_{e}=\rho^{\prime}-p^{\prime} / c_{\infty}^{2}
$$

is related to thermodynamic fluctuations. Primes in the above-given equations denote fluctuations around a steady flow state (see § 2.1).

One possible way to proceed is to solve for the acoustic pressure $p^{\prime}$ via numerical time integration of (3.1), simultaneously with a DNS of the near field, from which the right-hand-side terms are evaluated at each time step. The individual contribution of each source term to the acoustic far field can then be examined separately. This strategy has been applied, for instance, by Freund (2001) and Boersma (2005). Alternatively, a solution for the acoustic pressure field can be sought in terms of a Green's function to the wave operator, which is to be evaluated for the source terms of the Lighthill equation (3.1). The latter approach has been applied to the present jet configuration in a preliminary study (chapter 5 in Lesshafft 2006). The results clearly identify the excess density as the dominant acoustic source, and the sound field computed from this source term satisfactorily reproduces the directivity pattern displayed in figure 2 .

However, the interpretation of the results in Lesshafft (2006) remains inconclusive. First, the analysis shows that the excess density formally produces a monopole source distribution. The overall dipole character of the directly computed far field can still be retrieved, but the process requires a very accurate representation of phase variations in the source distribution, and therefore it is quite susceptible to numerical imprecisions. As a result, the extinction angle (near $\vartheta=90^{\circ}$ in figure 2) in the Lighthill analysis of Lesshafft (2006) is shifted by $15^{\circ}$ when compared with the simulation results. Second, it is difficult to interpret the physical nature of the sound-generating mechanisms represented by the excess density. Formally, $\rho_{e}=\rho^{\prime}-p^{\prime} / c_{\infty}^{2}$ appears to represent non-isentropic fluctuations, but this interpretation does not apply to flows with variable speed of sound, such as hot jets. For a better characterization of the relevant acoustic source mechanism in the case on hand, the observed sound radiation must be associated with a component of the excess density that explicitly produces a dipole field.

The following investigation will be based on an alternative formulation of (3.1), derived by Lilley $(1974,1996)$. By combining the momentum and energy equations (instead of using the momentum and continuity equations, as done by Lighthill 1952), Lilley obtained a wave equation for pressure fluctuations, equivalent to (3.1), where 
the excess-density source term is replaced by

$$
\frac{\partial^{2} \rho_{e}}{\partial t^{2}}=\frac{1}{c_{\infty}^{2}} \frac{\partial^{2}}{\partial t^{2}} K(\boldsymbol{x}, t)-\frac{1}{c_{\infty}} \frac{\partial^{2}}{\partial t \partial x_{i}} H_{i}(\boldsymbol{x}, t)-\frac{1}{c_{\infty}} \frac{\partial^{2}}{\partial t \partial x_{i}} D_{i}(\boldsymbol{x}, t) .
$$

Note that this is not what is commonly known as 'Lilley's equation' (derived in the same 1974 publication), which involves third-order derivatives and provides an improved separation of sound generation and propagation effects. The individual source terms in (3.4) are

$$
\begin{aligned}
K(\boldsymbol{x}, t) & =\frac{\gamma-1}{2} \rho|\boldsymbol{u}|^{2}, \\
H_{i}(\boldsymbol{x}, t) & =\frac{\gamma-1}{c_{\infty}} \rho u_{i}\left(h_{\infty}-h_{s}\right), \\
D_{i}(\boldsymbol{x}, t) & =\frac{\gamma-1}{c_{\infty}}\left(\tau_{i j} u_{j}-q_{i}\right) .
\end{aligned}
$$

The first two components, $K$ and $H_{i}$, represent fluctuations of the kinetic energy and of the total enthalpy flux, respectively. All diffusive effects, associated with $\tau$ and the heat flux $\boldsymbol{q}=-\nabla T /\left[(\gamma-1) M a_{c}^{2} \operatorname{RePr}\right]$, are contained in $D_{i}$. The stagnation enthalpy is defined as $h_{s}=h+|\boldsymbol{u}|^{2} / 2$, with the local enthalpy $h$ (see Lilley 1996). The far-field enthalpy is found as $h_{\infty}=c_{\infty}^{2} /(\gamma-1)$. In terms of conservative variables, as used in the present simulations (see $\S 2.1$ ), expression (3.6) can be rewritten as

$$
H_{i}(\boldsymbol{x}, t)=c_{\infty} \rho u_{i}-\frac{\gamma-1}{c_{\infty}}(\rho E+p) u_{i} .
$$

The spatial derivatives in (3.4) characterize the kinetic energy term as a monopole source, whereas the enthalpy- and diffusion-related terms are dipole sources. With respect to generalized acoustic analogy formulations in the recent literature, the dipole sources in (3.4) may be recovered as special cases of the source terms identified by Goldstein (2003, the $\eta_{i}^{\prime}$ term in 3.5 in his work) and by Morfey \& Wright (2007, the $p_{i j}$ term in 3.22 in their work).

\subsection{Far-field solution}

The Lighthill equation (3.1) as well as Lilley's decomposition (3.4) follow from exact manipulations of the governing flow equations. An approximate solution for pressure fluctuations in the far field is given by Lilley (1996) and may be rewritten in our notation as

$$
\begin{aligned}
p^{\prime}(\boldsymbol{\xi}, t)= & \frac{1}{4 \pi \xi c_{\infty}^{2}} \frac{\xi_{i} \xi_{j}}{\xi^{2}} \frac{\partial^{2}}{\partial t^{2}} \int \boldsymbol{S}_{i j}\left(\boldsymbol{x}, t^{\prime}\right) \mathrm{d}^{3} \boldsymbol{x}-\frac{1}{4 \pi \xi c_{\infty}^{2}} \frac{\partial^{2}}{\partial t^{2}} \int K\left(\boldsymbol{x}, t^{\prime}\right) \mathrm{d}^{3} \boldsymbol{x} \\
& -\frac{1}{4 \pi \xi c_{\infty}^{2}} \frac{\xi_{i}}{\xi} \frac{\partial^{2}}{\partial t^{2}} \int H_{i}\left(\boldsymbol{x}, t^{\prime}\right) \mathrm{d}^{3} \boldsymbol{x}-\frac{1}{4 \pi \xi c_{\infty}^{2}} \frac{\xi_{i}}{\xi} \frac{\partial^{2}}{\partial t^{2}} \int D_{i}\left(\boldsymbol{x}, t^{\prime}\right) \mathrm{d}^{3} \boldsymbol{x} .
\end{aligned}
$$

The acoustic signal observed at the far-field location $\xi$ at time $t$ has been emitted from location $x$ in the source region at the retarded time

$$
t^{\prime}=t-\frac{|\boldsymbol{x}-\boldsymbol{\xi}|}{c_{\infty}} \approx t-\frac{\xi}{c_{\infty}}+\frac{\boldsymbol{x} \cdot \boldsymbol{\xi}}{\xi c_{\infty}} .
$$

With approximation (3.10), and with the temporal and spatio-temporal Fourier transforms defined as

$$
\hat{f}(\boldsymbol{x}, \omega)=\int f(\boldsymbol{x}, t) \mathrm{e}^{\mathrm{i} \omega t} \mathrm{~d} t, \quad \tilde{f}(\boldsymbol{k}, \omega)=\iint f(\boldsymbol{x}, t) \mathrm{e}^{\mathrm{i}(\omega t-\boldsymbol{k} \cdot \boldsymbol{x})} \mathrm{d}^{3} \boldsymbol{x} \mathrm{d} t,
$$


the temporal Fourier-transformed acoustic pressure is found to be

$$
\begin{aligned}
\hat{p}(\xi, \omega) & =\frac{k_{a}^{2}}{4 \pi \xi} \mathrm{e}^{\mathrm{i} k_{a} \xi} \int\left\{-\frac{\xi_{i} \xi_{j}}{\xi^{2}} \hat{\boldsymbol{S}}_{i j}(\boldsymbol{x}, \omega)+\hat{K}(\boldsymbol{x}, \omega)+\frac{\xi_{i}}{\xi} \hat{H}_{i}(\boldsymbol{x}, \omega)+\frac{\xi_{i}}{\xi} \hat{D}_{i}(\boldsymbol{x}, \omega)\right\} \mathrm{e}^{-\mathrm{i} \boldsymbol{k}_{a} \cdot \boldsymbol{x}} \mathrm{d}^{3} \boldsymbol{x} \\
& =\frac{k_{a}^{2}}{4 \pi \xi} \mathrm{e}^{\mathrm{i} k_{a} \xi}\left[-\frac{\xi_{i} \xi_{j}}{\xi^{2}} \hat{\boldsymbol{S}}_{i j}\left(\boldsymbol{k}_{a}, \omega\right)+\tilde{K}\left(\boldsymbol{k}_{a}, \omega\right)+\frac{\xi_{i}}{\xi} \tilde{H}_{i}\left(\boldsymbol{k}_{a}, \omega\right)+\frac{\xi_{i}}{\xi} \tilde{D}_{i}\left(\boldsymbol{k}_{a}, \omega\right)\right] .
\end{aligned}
$$

The acoustic wave vector is defined as $\boldsymbol{k}_{a}=\omega / c_{\infty} \boldsymbol{e}_{\xi}$, oriented in the observer direction. As pointed out by Crighton (1975), the acoustic radiation observed at a given far-field location $\boldsymbol{\xi}$ is caused by a single spectral component of the source distribution, namely a plane wave of wavenumber $k_{a}$, travelling in the radiation direction.

\subsection{Axisymmetric and radially compact sources}

\subsubsection{Axial symmetry}

Under the assumption of an axisymmetric source term distribution consisting only of $S_{i j}$, (3.13) has been rewritten by Huerre \& Crighton (1983) for the particular case of a Gaussian envelope function $S_{i j}(r, x) \propto \exp \left(-x^{2} / \sigma^{2}\right)$. Fleury (2006) gives a more general formulation, valid for arbitrary envelope shapes, and the excess-density source term (3.1) has been included in Lesshafft (2006).

In order to evaluate the spatial Fourier integrals in (3.12), source locations $\boldsymbol{x}$ are expressed in cylindrical coordinates $(x, r, \varphi)$, while the observer location $\xi$ in the axisymmetric far field is characterized by its spherical coordinates $(\xi, \vartheta)$. The resulting integral can be solved numerically in all three $(x, r, \varphi)$ directions, as done by Mitchell et al. (1999). However, Huerre \& Crighton (1983) have noted that the azimuthal integration admits closed-form solutions in terms of Bessel functions. After integration in $\varphi,(3.13)$ becomes

$$
\hat{p}(\xi, \vartheta, \omega)=\frac{k_{a}^{2}}{2 \xi} \mathrm{e}^{\mathrm{i} k_{a} \xi} \iint D(x, r, \vartheta, \omega) \mathrm{e}^{-\mathrm{i} k_{a} x \cos \vartheta} r \mathrm{~d} r \mathrm{~d} x,
$$

with the integrand given by

$$
\begin{aligned}
D(x, r, \vartheta, \omega) & =I_{S x x}+I_{S r x}+I_{S r r}+I_{S \varphi \varphi}+I_{K}+I_{H x}+I_{H r}+I_{D x}+I_{D r}, \\
I_{S x x} & =-\mathrm{J}_{0}(\alpha r) \cos ^{2} \vartheta \hat{S}_{x x}(x, r, \omega), \\
I_{S r x} & =-\mathrm{i}_{1} \mathrm{~J}_{1}(\alpha r) \sin \vartheta \cos \vartheta \hat{S}_{r x}(x, r, \omega), \\
I_{S r r} & =-0.5\left[\mathrm{~J}_{0}(\alpha r)-\mathrm{J}_{2}(\alpha r)\right] \sin ^{2} \vartheta \hat{S}_{r r}(x, r, \omega), \\
I_{S \varphi \varphi} & =-0.5\left[\mathrm{~J}_{0}(\alpha r)+\mathrm{J}_{2}(\alpha r)\right] \sin ^{2} \vartheta \hat{S}_{\varphi \varphi}(x, r, \omega), \\
I_{K} & =\mathrm{J}_{0}(\alpha r) \hat{K}(x, r, \omega), \\
I_{H x} & =\mathrm{J}_{0}(\alpha r) \cos \vartheta \hat{H}_{x}(x, r, \omega), \\
I_{H r} & =\mathrm{iJ}_{1}(\alpha r) \sin \vartheta \hat{H}_{r}(x, r, \omega), \\
I_{D x} & =\mathrm{J}_{0}(\alpha r) \cos \vartheta \hat{D}_{x}(x, r, \omega), \\
I_{D r} & =\mathrm{iJ}_{1}(\alpha r) \sin \vartheta \hat{D}_{r}(x, r, \omega) .
\end{aligned}
$$

The argument of the Bessel functions $\mathrm{J}_{i}$ is $\alpha r=-k_{a} r \sin \vartheta$. Equation (3.14) explicitly gives the far-field pressure in terms of a spatial phase, a radial decay $\propto \xi^{-1}$ and a $\vartheta$-dependent far-field directivity function. The Bessel functions represent the effect of azimuthal interference, whereas factors composed of $\cos \vartheta$ and $\sin \vartheta$ produce quadrupole or dipole directivities. Each source term contains an 'antenna factor', which depends on the spatial distribution $\hat{S}_{x x}(r, x, \omega), \ldots, \hat{D}_{r}(r, x, \omega)$ of the source strength. 
The influence of the antenna factor on the directivity pattern is commonly referred to as the superdirective effect, following the interpretation given by Crighton \& Huerre (1990) of the experimental results of Laufer \& Yen (1983, see $§ 1$ ). For an analysis of the acoustic radiation from the present jet configuration, discussed in $\S 2.2$, the distributions $\hat{S}_{x x}(r, x, \omega), \ldots, \hat{D}_{r}(r, x, \omega)$ may be obtained directly from the numerical simulation.

\subsubsection{Radial compactness}

Following the approach of Huerre \& Crighton (1983), the aeroacoustic source distributions in a jet may be assumed to be compact in the radial direction but not necessarily so in the axial direction. For the present analysis, the source terms are modelled as being concentrated in the centre of the shear layer at $r=1$ such that for instance

$$
\hat{S}_{i j}(x, r, \omega)=\hat{S}_{i j}^{x}(x, \omega) \delta(r-1),
$$

and therefore

$$
\begin{aligned}
& \hat{S}_{i j}^{x}(x, \omega)=\int_{0}^{\infty} \hat{S}_{i j}(x, r, \omega) r \mathrm{~d} r, \\
& \tilde{S}_{i j}^{x}(k, \omega)=\int_{-\infty}^{\infty} \hat{S}_{i j}^{x}(x, \omega) \mathrm{e}^{-\mathrm{i} k x} \mathrm{~d} x .
\end{aligned}
$$

These integrals must be solved numerically. Radially compact representations of all other source terms and their Fourier transforms, marked by the superscript $x$ in the following, are obtained accordingly.

Under the assumption of radial compactness of all aeroacoustic sources, (3.14) simplifies to

$$
\hat{p}(\xi, \vartheta, \omega)=\frac{k_{a}^{2}}{2 \xi} \mathrm{e}^{\mathrm{i} k_{a} \xi} D^{x}(\vartheta, \omega),
$$

with

$$
\begin{aligned}
D^{x}(\vartheta, \omega) & =I_{S x x}^{x}+I_{S r x}^{x}+I_{S r r}^{x}+I_{S \varphi \varphi}^{x}+I_{K}^{x}+I_{H x}^{x}+I_{H r}^{x}+I_{D x}^{x}+I_{D r}^{x}, \\
I_{S x x}^{x} & =-\mathrm{J}_{0}(\alpha) \cos ^{2} \vartheta \tilde{S}_{x x}^{x}\left(k_{a} \cos \vartheta, \omega\right), \\
I_{S x}^{x} & =-\mathrm{i} 2 \mathrm{~J}_{1}(\alpha) \sin \vartheta \cos \vartheta \tilde{S}_{r x}^{x}\left(k_{a} \cos \vartheta, \omega\right), \\
I_{S r}^{x} & =-0.5\left[\mathrm{~J}_{0}(\alpha)-\mathrm{J}_{2}(\alpha)\right] \sin ^{2} \vartheta \tilde{S}_{r r}^{x}\left(k_{a} \cos \vartheta, \omega\right), \\
I_{S \varphi \varphi}^{x} & =-0.5\left[\mathrm{~J}_{0}(\alpha)+\mathrm{J}_{2}(\alpha)\right] \sin ^{2} \vartheta \tilde{S}_{\varphi \varphi}^{x}\left(k_{a} \cos \vartheta, \omega\right), \\
I_{K}^{x} & =\mathrm{J}_{0}(\alpha) \tilde{K}^{x}\left(k_{a} \cos \vartheta, \omega\right), \\
I_{H x}^{x} & =\mathrm{J}_{0}(\alpha) \cos \vartheta \tilde{H}_{x}^{x}\left(k_{a} \cos \vartheta, \omega\right), \\
I_{H r}^{x} & =\mathrm{iJ}_{1}(\alpha) \sin \vartheta \tilde{H}_{r}^{x}\left(k_{a} \cos \vartheta, \omega\right), \\
I_{D x}^{x} & =\mathrm{J}_{0}(\alpha) \cos \vartheta \tilde{D}_{x}^{x}\left(k_{a} \cos \vartheta, \omega\right), \\
I_{D r}^{x} & =\mathrm{iJ}_{1}(\alpha) \sin \vartheta \tilde{D}_{r}^{x}\left(k_{a} \cos \vartheta, \omega\right) .
\end{aligned}
$$

The Bessel functions now take the argument $\alpha=-k_{a} \sin \vartheta$. At low Mach numbers, where $k_{a} \ll 1$, variations of the Bessel functions are negligible. A factor $\mathrm{J}_{0}(\alpha) \approx 1$ is associated with source terms that are nearly unaffected by azimuthal interference, whereas a factor $J_{1}(\alpha) \approx 0$ signifies almost complete cancellation of a source with its image across the jet axis. The antenna factor owing to each source term distribution is given explicitly by its one-dimensional Fourier transform along $x$. From the argument 

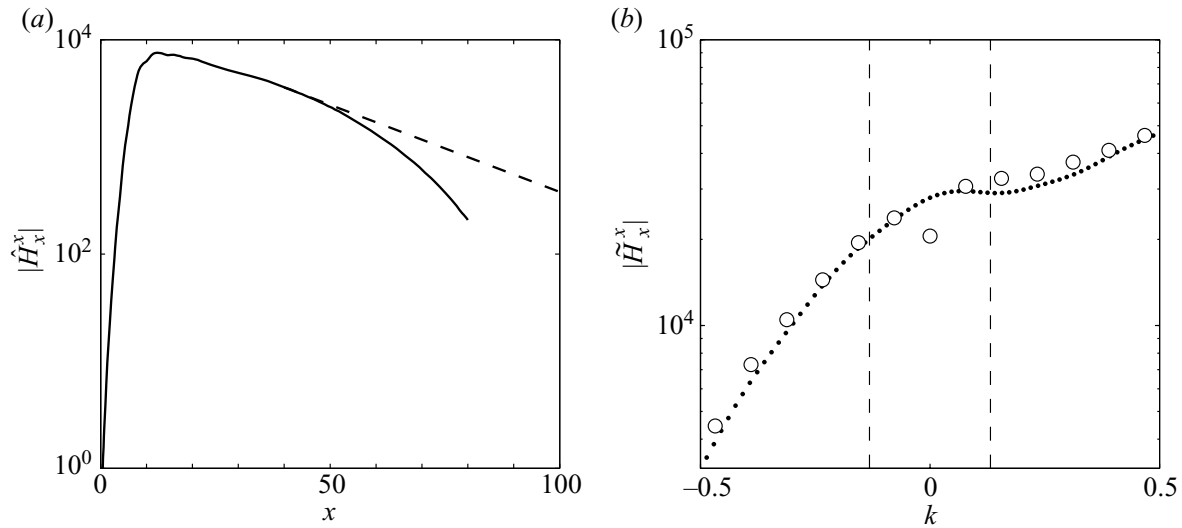

FiguRE 4. (a) Jet with $S=0.3$ : amplitude of the radially compact enthalpy-flux source term $\left|\hat{H}_{x}^{x}\right|$ as a function of $x$. The solid line denotes original DNS data; the dashed line denotes extrapolated exponential decay over the interval $40 \leqslant x \leqslant 400$. (b) Corresponding discrete Fourier spectra near $k=0$; the white circles are based on original DNS data; the black dots are based on the extrapolated amplitude envelope. The dashed lines indicate the acoustically relevant interval $-k_{a} \leqslant k \leqslant k_{a}$.

$k=k_{a} \cos \vartheta$, it is seen again that only the interval $-k_{a} \leqslant k \leqslant k_{a}$ of the spectrum radiates sound into the far field.

\section{Acoustic source term analysis for hot jets}

\subsection{Marginal globally unstable case $S=0.3$}

The far-field pressure solution (3.28) is first evaluated for the jet configuration with $S=0.3$. All source term distributions $\hat{S}_{i j}, \hat{K}, \hat{H}_{i}$ and $\hat{D}_{i}$, as defined in (3.2), (3.5)(3.7) and (3.11), are extracted from the DNS as functions of $r$ and $x$. The temporal Fourier transform (3.11) of all sources is evaluated for $\omega=\omega_{g}$ during runtime over one cycle period. Under the assumption of radial compactness, the one-dimensional distributions $\hat{S}_{i j}^{x}, \hat{K}^{x}, \hat{H}_{i}^{x}$ and $\hat{D}_{i}^{x}$ are obtained according to (3.26) by numerical integration in $r$. Evaluation of the far-field pressure solution (3.28) then involves a discrete Fourier transform in $x$.

As an example, the streamwise variation of the enthalpy-flux source envelope $\left|\hat{H}_{x}^{x}\left(x, \omega_{g}\right)\right|$ is presented in figure $4(a)$ as a solid line. It displays a sharp front near the upstream boundary and a slow decay downstream of the vortex roll-up location around $x=9$. Accelerated decay is observed near the end of the numerical domain, but the source amplitude at the downstream end $x=80$ is still two orders of magnitude larger than at the upstream boundary. As a result, the truncation of the spatial domain may produce significant errors in the spatial Fourier transform. The corresponding discrete spectrum $\left|\tilde{H}_{x}^{x}\left(k, \omega_{g}\right)\right|$ near $k=0$ is displayed in figure $4(b)$ as the white circles. Its peak, not shown in the diagram, is found at $k_{\max }=1.28$, with a value of $\left|\tilde{H}_{x}^{x}\left(k_{\max }, \omega_{g}\right)\right|=2.5 \times 10^{6}$. Since the streamwise physical extent of the numerical domain, $0 \leqslant x \leqslant 80$, is less than twice the acoustic wavelength, only three points of the spectrum can be obtained within the radiating interval $-k_{a} \leqslant k \leqslant k_{a}$. A discussion of the directivity pattern based on only three data points is unsatisfactory; it is therefore desirable to first achieve a higher spectral resolution of the spatial Fourier transform. 


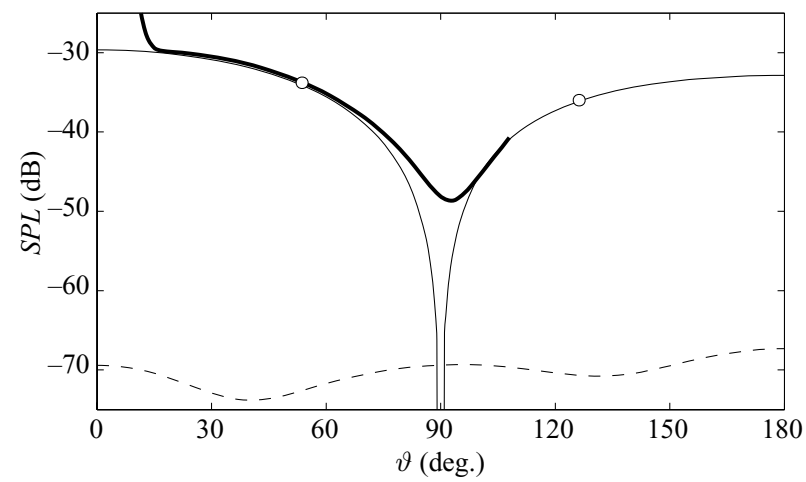

FIGURE 5. Jet with $S=0.3$ : directivity of the acoustic far field, comparison between DNS and Lighthill solution; - , directly computed sound; $\bigcirc$, Lighthill solution owing to $H_{x}^{x}$ without streamwise extrapolation. The Lighthill solution from extrapolated sources: - enthalpy-flux term $H_{x}^{x}$ alone; - - -, combined radiation from all other terms.

Mitchell et al. (1999) faced the same problem in their computations of the acoustic field in forced isothermal jets. These authors suggested to extrapolate the source term distributions, prior to evaluating the Lighthill solution, over a sufficiently long downstream region beyond the end of the computational domain. Following this idea, the source terms in the present calculations are taken to develop exponentially as

$$
f(x)=f\left(x_{0}\right) \mathrm{e}^{\mathrm{i} k_{x}\left(x-x_{0}\right)} \quad \text { with } \quad k_{x}=-\mathrm{i} \frac{f^{\prime}\left(x_{0}\right)}{f\left(x_{0}\right)},
$$

from $x_{0}=40$ down to $x=400$. The extrapolated source envelope $\left|\hat{H}_{x}^{x}\left(x, \omega_{g}\right)\right|$ is shown as a dashed line in figure $4(a)$. Over the interval $20 \leqslant x \leqslant 50$, the amplitude decay of the original distribution may indeed be well approximated by an exponential function. The black dots in figure $4(b)$ represent the discrete Fourier spectrum $\left|\tilde{H}_{x}^{x}\left(k, \omega_{g}\right)\right|$ of the extrapolated source distribution. They align well with the previously obtained data points (white circles) in the vicinity of the radiating interval, which now is resolved by 17 points. In particular, the extrapolation procedure is found to appropriately correct the apparent error, due to domain truncation, in the $k=0$ component of the original spectrum.

The Lighthill solution obtained from extrapolated source distributions in the $S=0.3$ jet, according to (3.28), is compared with the DNS results in figure 5. The thick line represents directly computed sound pressure levels, measured along the arc in figure 1. At angles less than $18^{\circ}$, the arc crosses the hydrodynamic near field. The thin line interpolates the Lighthill solution owing to the extrapolated $H_{x}^{x}$ term, whereas results based on the non-extrapolated $H_{x}^{x}$ distribution are shown as the white circles. The combined acoustic radiation from all other source terms, as obtained from (3.28), is represented by a dashed line. Sound pressure levels are computed as $S P L=20 \log _{10}\left(\xi|\hat{p}| / p_{\text {ref }}\right)$, thereby eliminating the $\xi^{-1}$ decay of the acoustic pressure amplitude. The decibel scaling takes the near-field pressure maximum $p_{\text {ref }}=\max _{\boldsymbol{x}}\left|\hat{p}\left(\boldsymbol{x}, \omega_{g}\right)\right|$ as a common reference value for all curves.

Figure 5 demonstrates that the dipole strength of the directly computed acoustic field is very well retrieved by the Lighthill prediction for dipole radiation due to the axial enthalpy-flux term $H_{x}^{x}$. Over the interval $20^{\circ} \leqslant \vartheta \leqslant 60^{\circ}$, both curves coincide within $0.5 \mathrm{~dB}$ accuracy. Without source extrapolation, the one point obtained within the downstream lobe, at $\vartheta=54^{\circ}$, matches the simulation result with a precision of 
(a)

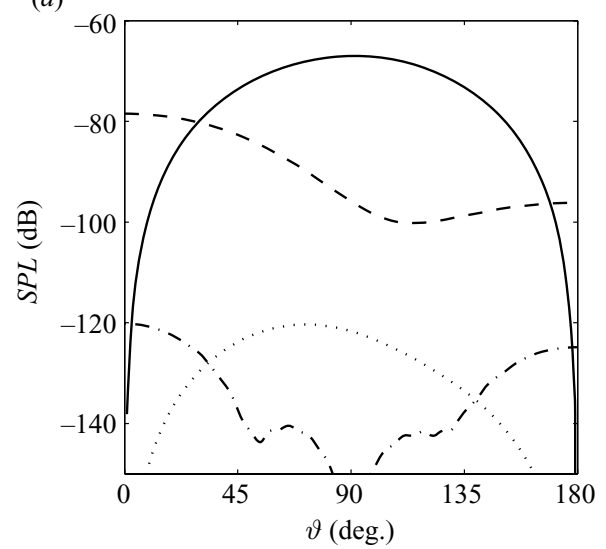

(b)

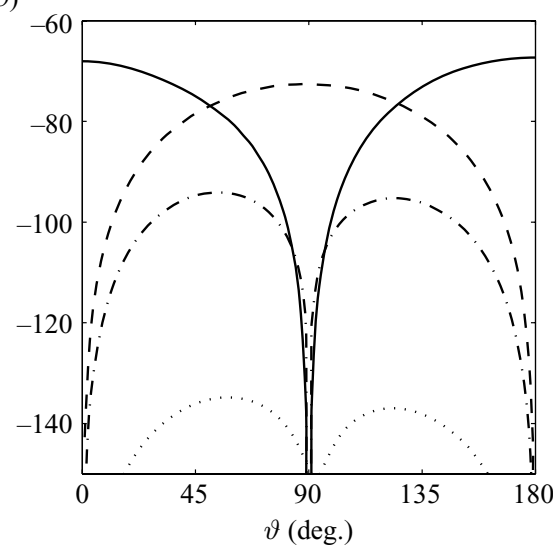

FiguRE 6. Jet with $S=0.3$ : individual far-field contributions from all source terms except $H_{x}^{x}$. (a) Excess-density components: -,$H_{r}^{x} ;-\cdots, K^{x} ;-\cdots, D_{x}^{x} ; \cdots, D_{r}^{x}$. (b) Reynolds and viscous stresses: -,$S_{x x}^{x} ;-\cdots, S_{r r}^{x} ;-\cdots, S_{r x}^{x} ; \cdots, S_{\varphi \varphi}^{x}$.

$0.1 \mathrm{~dB}$. Furthermore, the separation of individual source term contributions clearly indicates that the radiation due to $H_{x}^{x}$ strongly dominates the acoustic field in the present jet configuration: the combined radiation from all other aeroacoustic sources together does not exceed $-68 \mathrm{~dB}$ at any angle. Near $\vartheta=90^{\circ}$, where this low-level radiation may in principle be detected, the far-field simulation results are likely to be affected by spurious numerical effects, most notably due to unphysical acoustic reflections at the domain boundaries.

The results presented in figure 5 are quite robust with respect to details of the extrapolation (4.1). A choice of $x_{0}$ anywhere in the interval $20 \leqslant x \leqslant 60$ locally changes the $S P L$ values associated with $H_{x}^{x}$ by not more than $0.5 \mathrm{~dB}$.

Individual contributions of all other source terms are documented in figure 6 . Acoustic emission from dissipative effects $\left(D_{x}^{x}\right.$ and $D_{r}^{x}$, the dash-dotted and dotted lines in figure $6 a$ ) scales with the Reynolds number, and in the present case it is found to be negligible even when compared with the low-level radiation from the radial enthalpy flux $H_{r}^{x}$ and the kinetic energy fluctuations $K^{x}$. While the fluctuation amplitude of $H_{r}^{x}$ in the jet is comparable to that of the axial enthalpy flux $H_{x}^{x}$, its acoustic radiation is much less significant in an axisymmetric setting, because of the azimuthal interference given by $J_{1}(\alpha)$ in (3.36). Acoustic radiation from Reynolds stresses $S_{x x}^{x}$ and $S_{r r}^{x}$ (the solid and dashed lines in figure 6b) is similar in strength to that from $H_{r}^{x}$ and $K^{x}$, while the effects of $S_{r x}^{x}$ and $S_{\varphi \varphi}^{x}$ are negligible. Although viscous stresses are included in the $S_{i j}$ terms (3.2), their contribution is found to be insignificant.

The Lighthill solution in figure 5 indicates a difference of only $3 \mathrm{~dB}$ between the upstream and downstream acoustic amplitudes. This difference characterizes the weak superdirective quality of the acoustic field; it is caused by the antenna factor, i.e. by the variation of spectral density $\tilde{H}_{x}^{x}$ between $-k_{a}$ and $+k_{a}$. In comparison with the beam-like directivity pattern (1.1) measured by Laufer \& Yen (1983), superdirectivity in the present configuration can be said to be negligible.

According to Crighton \& Huerre (1990), superdirective radiation in the particular form of (1.1) would occur if the spectrum in figure $4(b)$ took the shape of a narrow Gauß function, centred at the carrier wavenumber $k_{\max }=1.28$ and extending down to 


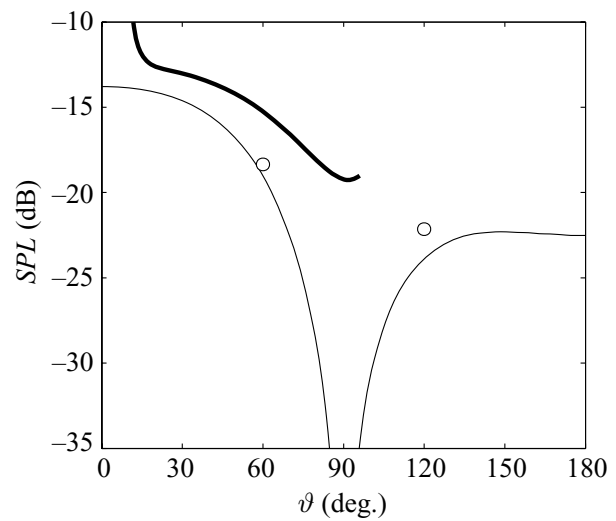

(a) $S=0.1$

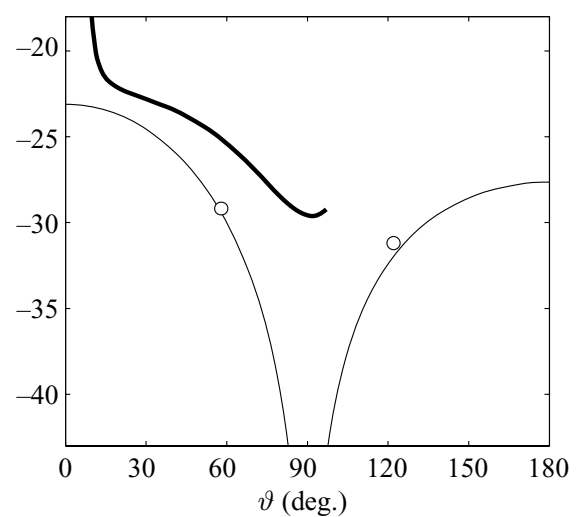

(b) $S=0.2$

FIGURE 7. Supercritically hot jets with $(a) S=0.1$, (b) $S=0.2$. Directivity of the acoustic far field and comparison between DNS and the Lighthill solution: directly computed sound (-); Lighthill solution owing to enthalpy flux source term $H_{x}^{x}$ without extrapolation $(\bigcirc)$ and with extrapolation (-). Other sources are negligible (see the text).

the acoustic range $|k|<k_{a}$. In the low-Mach-number limit $\left(k_{\max } \gg k_{a}\right)$, this assumption indeed necessitates a perfectly Gaussian source distribution $\left(\propto \exp \left(x^{2}\right)\right)$ in physical space over a large streamwise interval. Instead, it has been found that the source amplitude in the present configuration decays exponentially $(\propto \exp (x))$, as in the isothermal jet simulations of Mitchell et al. (1999). Crighton \& Huerre (1990) have demonstrated that an exponential source envelope cannot give rise to a superdirective factor as measured by Laufer \& Yen (1983).

A recent study by Obrist (forthcoming) has generalized the analysis of Crighton \& Huerre (1990) to two-dimensional wavepackets that are non-compact in both spatial directions. It is found that a finite cross-stream extent of the acoustic source region may strengthen the superdirective character of the far field. Whether the assumption of radial compactness is valid in the present context may be assessed by solving (3.14) with the two-dimensional source distribution $\hat{H}_{x}\left(r, x, \omega_{g}\right)$. It is difficult to define a consistent extrapolation procedure in two dimensions; therefore the non-compact solution may only be computed for radiation angles $\vartheta=54^{\circ}$ and $126^{\circ}$. At both angles, the results are found to match the compact solution (the white circles in figure 5) within $0.005 \mathrm{~dB}$ accuracy.

\subsection{Supercritically heated jets: $S=0.1$ and $S=0.2$}

The analysis carried out for the $S=0.3$ jet in the preceding section is now applied to the configurations with $S=0.1$ and 0.2 (see $\S 2.1$ ). The global frequencies, as reported in Lesshafft et al. (2006), are $\omega_{g}=0.493$ and 0.658 , respectively, and the acoustic wavelengths therefore are $\lambda_{a}=40.3$ and 42.7 . The main results of the analysis are shown in figure 7 : the thick lines trace the acoustic directivity measured in the far field of the direct numerical simulations, and the thin lines and the circles represent the Lighthill solution owing to the axial enthalpy flux source term $H_{x}^{x}$ with and without streamwise extrapolation. As in the $S=0.3$ jet, acoustic emission from all other source terms is found to be negligible in comparison. Their combined radiation, not shown in figure 7 , is below $-45 \mathrm{~dB}(S=0.1)$ and $-64 \mathrm{~dB}(S=0.2)$. Sound pressure levels in both cases are again scaled with the respective maximum values of $\left|\hat{p}\left(\boldsymbol{x}, \omega_{g}\right)\right|$ in the near field. 
Agreement between the directly computed acoustic field and the Lighthill solution for these two configurations is less accurate than in the $S=0.3$ case. In both parts of figure 7 , simulation and prediction results are separated by almost $2 \mathrm{~dB}$ at $\vartheta=20^{\circ}$ and $5 \mathrm{~dB}$ at $\vartheta=60^{\circ}$. However, the overall dipole character is well retrieved in both cases, and the agreement may be regarded as sufficiently close in order to allow for a qualitative discussion. It can therefore be concluded that axial enthalpy flux fluctuations are the dominant acoustic source mechanism in globally unstable hot jets also at temperature ratios $S=0.1$ and 0.2 . The resulting acoustic field is that of a dipole, with maximum intensity in the upstream and downstream directions. Amplitude modulations due to the axial non-compactness of the source distribution are found to be moderately small, and the apparent source location corresponds to the region of vortex roll-up $(x=3$ for $S=0.1$ and $x=4$ for $S=0.2)$. It is noted again that the acoustic radiation due to vortex pairing, as a secondary instability of the global mode structure, is not considered in the present study.

DNS results for the supercritical cases $S=0.1$ and 0.2 are much more affected by numerical inaccuracies than the marginally unstable case $S=0.3$. These inaccuracies stem primarily from the numerical treatment of the upstream boundary conditions (see $§ 2.1$ ). As discussed in Lesshafft et al. $(2006,2007)$, the location of vortex rollup moves closer to the upstream domain boundary as the flow becomes more supercritical with decreasing values of $S$. This results in stronger spurious reflections because the acoustic waves now propagate almost parallel to the upstream boundary, whereas the numerical treatment assumes nearly perpendicular incidence of acoustic waves. Spurious reflections increase the acoustic energy contained in the numerical domain. Unphysical interaction between acoustic and vortical perturbations at the upstream boundary must also be expected to increase as perturbation amplitudes reach nonlinear levels close to the inlet. It has been noted in Lesshafft et al. (2006, 2007) that the influence of the numerical boundary treatment on the near-field dynamics is stronger for low values of $S$.

\section{Conclusion}

The near and far fields of three globally unstable hot jets have been resolved in direct numerical simulations of the axisymmetric Navier-Stokes equations. The three configurations have temperature ratios $S=0.1,0.2$ and 0.3 and associated Mach number values $M a_{\infty}=0.32,0.22$ and 0.18 . Results have been discussed in depth for the marginally unstable configuration with temperature ratio $S=0.3$ : the nonlinear global mode in the near field, described by Lesshafft et al. (2006), radiates into the far field as a compact acoustic dipole, with maximum amplitude along the jet axis. A solution to the Lighthill equation, including source terms associated with the fluctuations of enthalpy flux, kinetic energy, Reynolds stresses and dissipative effects (Lilley 1974, 1996), has been formulated for an axisymmetric geometry, with and without the assumption of radially compact source distributions. In order to retrieve the directly computed far-field directivity pattern, the source distributions used in the Lighthill analysis have been extrapolated far beyond the downstream boundary of the computational domain used in the simulation. For the reference configuration with temperature ratio $S=0.3$, the resulting Lighthill solution matches the directly computed acoustic field within $0.5 \mathrm{~dB}$ accuracy over a large range of the radiation angle. The decomposition of source terms has demonstrated that the global mode acoustic field is strongly dominated by dipole radiation due to axial enthalpy flux fluctuations. 
The Lighthill analysis has been extended to the supercritical regime of strongly heated jets, with temperature ratios $S=0.1$ and 0.2 . Although the numerical simulation data obtained for these two configurations are not of the same high quality as in the $S=0.3$ case, and the agreement with the Lighthill solution for the acoustic field is less accurate as a consequence, the analysis supports the same principal conclusion: the source mechanism related to the axial enthalpy flux strongly dominates the overall acoustic radiation, giving rise to a dipole directivity in the far field. Comparison between figures 5 and 7 demonstrates that the acoustic intensity increases with stronger heating.

Antenna effects, which could potentially yield a superdirective radiation pattern, are not found to be significant in the present configurations. At $S=0.3$, the antenna factor only causes a $3 \mathrm{~dB}$ difference between the $\vartheta=0^{\circ}$ and $\vartheta=180^{\circ}$ directions. The absence of superdirectivity is due to the spatial shape of the global mode wave packet: its $k$-spectrum is very different from a Gaussian shape (see figure $4 b$ ), and it presents only weak variations over the radiating interval $-k_{a} \leqslant k \leqslant k_{a}$. Higher values of the Mach number will probably favour superdirectivity; if $M a$ is increased, the radiating window is enlarged and may contain regions of large variations in spectral density. An extension of the present study to externally forced jets in the high-subsonic-Mach-number regime will be the focus of future investigations.

It is a pleasure to dedicate this study to Steve Davis in recognition of his many fundamental contributions to fluid mechanics. Steve is a source of inspiration to all of us, from both a scientific and a personal point of view. We are very grateful to Professor Marvin Goldstein for his suggestion to employ Lilley's source term decomposition in the present study. Financial support for this work was provided by ONERA and by the EADS Foundation.

\section{REFERENCES}

ANDERSSON, N., ERIKSSON, L. E. \& DAVIDSON, L. 2005 Large-eddy simulation of subsonic turbulent jets and their radiated sound. AIAA J. 43 (9), 1899-1912.

Bodony, D. J. \& Lele, S. K. 2005 On using large-eddy simulation for the prediction of noise from cold and heated turbulent jets. Phys. Fluids 17, 085103.

Bodony, D. J. \& LeLE, S. K. 2006 Review of the current status of jet noise predictions using large-eddy simulation. Paper 2006-0468. AIAA.

Bodony, D. J. \& LeLE, S. K. 2008 On using large-eddy simulation for the prediction of noise from cold and heated turbulent jets. J. Fluid Mech. 617, 231-253.

Boersma, B. 2005 Large eddy simulation of the sound field of a round turbulent jet. Theoret. Comput. Fluid Dyn. 19, 161-170.

Bogey, C. \& BAILly, C. 2004 Investigation of subsonic jet noise using LES: Mach and Reynolds number effects. Paper 2004-3023. AIAA.

Bogey, C. \& Bailly, C. 2005 Effects of inflow conditions and forcing on subsonic jet flows and noise. AIAA J. 43 (5), 1000-1007.

Bogey, C., BAilly, C. \& JuvÉ, D. 2003 Noise investigation of a high subsonic, moderate Reynolds number jet using a compressible large eddy simulation. Theoret. Comput. Fluid Dyn. 16, 273-297.

Crighton, D. 1975 Basic principles of aerodynamic noise generation. Progr. Aerosp. Sci. 16, 31-96.

Crighton, D. \& Huerre, P. 1990 Shear-layer pressure fluctuations and superdirective acoustic sources. J. Fluid Mech. 220, 355-368.

Dowling, A. 1992 Thermoacoustic sources and instabilities. In Modern Methods in Analytical Acoustics, pp. 378-405. Springer.

Fleury, V. 2006 Superdirectivité, bruit d'appariement et autres contributions au bruit de jet subsonique. PhD thesis, École Centrale de Lyon, Écully, France. 
Fleury, V., Bailly, C. \& JuvÉ, D. 2005 Shear-layer acoustic radiation in an excited subsonic jet: experimental study. C. R. Mec. 333, 746-753.

FortunÉ, V. \& Gervais, Y. 1999 Numerical investigation of the noise radiated from hot subsonic turbulent jets. AIAA J. 37 (9), 1055-1061.

Freund, J. 2001 Noise sources in a low-Reynolds-number turbulent jet at Mach 0.9. J. Fluid Mech. 438, 277-305.

Freund, J. 2003 Noise source turbulence statistics and the noise from a Mach 0.9 jet. Phys. Fluids 15 (6), 1788-1799.

GILES, M. 1990 Nonreflecting boundary conditions for Euler equation calculations. AIAA J. 28, 2050-2058.

Goldstein, M. E. 2003 A generalized acoustic analogy. J. Fluid Mech. 488, 315-333.

Huerre, P. \& Crighton, D. 1983 Sound generation by instability waves in a low Mach number flow. Paper 83-0661. AIAA.

Jordan, P., Gervais, Y., Valière, J.-C. \& Foulon, H. 2002 Results from acoustic field measurements. Tech Rep. G4RD-CT2000-00313. Laboratoire d'Etudes Aérodynamiques, Université de Poitiers.

Laufer, J. \& Yen, T.-C. 1983 Noise generation by a low-Mach-number jet. J. Fluid Mech. 134, 1-31.

LESSHAFFT, L. 2006 Nonlinear global modes and sound generation in hot jets. PhD thesis, École Polytechnique, Palaiseau, France.

Lesshafft, L. \& Huerre, P. 2007 Linear impulse response in hot round jets. Phys. Fluids 19, 024102.

Lesshafft, L., Huerre, P. \& SAgaut, P. 2007 Frequency selection in globally unstable round jets. Phys. Fluids 19, 054108.

Lesshafft, L., Huerre, P., Sagaut, P. \& Terracol, M. 2006 Nonlinear global modes in hot jets. J. Fluid Mech. 554, 393-409.

Lew, P.-T., Blaisdell, G. A. \& Lyrintzis, A. S. 2007 Investigation of noise sources in turbulent hot jets using large-eddy simulation data. Paper 2007-0016. AIAA.

Lighthill, M. 1952 On sound generated aerodynamically. Part I. General theory. Proc. R. Soc. Lond. A 201 (1107), 564-587.

LILLEY, G. M. 1974 On the noise from jets. AGARD-CP 131, 13.1-13.12.

LiLley, G. M. 1996 The radiated noise from isotropic turbulence with application to the theory of jet noise. J. Sound Vib. 190, 463-476.

Michalke, A. 1984 Survey on jet instability theory. Progr. Aerosp. Sci. 21, 159-199.

Mitchell, B., Lele, S. \& Moin, P. 1999 Direct computation of the sound generated by vortex pairing in an axisymmetric jet. J. Fluid Mech. 383, 113-142.

Monkewitz, P., Bechert, D., Barsikow, B. \& Lehmann, B. 1990 Self-excited oscillations and mixing in a heated round jet. J. Fluid Mech. 213, 611-639.

Morfey, C. L. \& Wright, M. 2007 Extensions of Lighthill's acoustic analogy with application to computational aeroacoustics. Proc. R. Soc. A 463, 2101-2127.

Obrist, D. 2009 Directivity of acoustic emissions from wave packets to the far-field. J. Fluid Mech. Forthcoming.

Shur, M., Spalart, P. R. \& Strelets, M. K. 2005 Noise prediction for increasingly complex jets. Part I. Methods and tests. Intl J. Aeroacous. 4 (3-4), 213-266.

Tanna, H. K. 1977 An experimental study of jet noise. Part I. Turbulent mixing noise. J. Sound Vib. 50 (3), 405-428.

Viswanathan, K. 2004 Aeroacoustics of hot jets. J. Fluid Mech. 516, 39-82.

WANG, M., Freund, J. B. \& Lele, S. K. 2006 Computational prediction of flow-generated sound. Annu. Rev. Fluid Mech. 38, 483-512. 